\title{
Embedding Intelligent Eco-aware Systems within Everyday Things to Increase People's Energy Awareness
}

\author{
Diego Casado-Mansilla* • Juan López-de-Armentia* • Daniela Ventura $†$ • \\ Pablo Garaizar* • Diego López-de-Ipiña*
}

Received: date / Accepted: date

\begin{abstract}
There is a lack of energy consumption awareness in working spaces. People in their workplaces do not receive energy consumption feedback nor do they pay a monthly invoice to electricity providers. In order to enhance workers' energy awareness, we have transformed everyday shared electrical appliances which are placed in common spaces (e.g. beamer projectors, coffeemakers, printers, screens, portable fans, kettles, and so on.) into persuasive eco-aware everyday things. The proposed approach lets these appliances report their usage patterns to a Cloud-server where the data is transformed into time-series and then processed to obtain the appliances' next-week usage forecast. Autoregressive Integrated Moving Average (ARIMA) model has been selected as the potentially most accurate method for processing such usage predictions when compared with the performance exhibited by three different configurations of Artificial Neural Networks (ANNs). Our major contribution is the application of soft computing techniques to the field of sustainable persuasive technologies. Thus, consumption predictions are used to trigger timely persuasive interactions to help devices users to operate the appliances as efficiently, energywise, as possible. Qualitative and quantitative results were gathered in a between-three-groups study related with the use of shared electrical coffee-makers at work-
\end{abstract}

\footnotetext{
* Authors

Deusto Institute of Technology - DeustoTech, University of Deusto

Avda. Universidades 24, 48007 - Bilbao, Spain

E-mail: \{dcasado, juan.lopezdearmentia, garaizar, and dipina\}@deusto.es

$\dagger$ Author

University of Catania - Italy

Dep. of Electrical, Electronic and Computer Engineering

E-mail: daniela.ventura@dieei.unict.it
}

place. The goal of these studies was to assess the effectiveness of the proposed eco-aware design in a workplace environment in terms of energy saving and the degree of affiliation between people and the smart appliances to create a green-team relationship.

Keywords Eco-aware everyday things - Persuasive Eco-feedback · Energy awareness · Machine learning · Time Series · ARIMA models

\section{Introduction}

Methodologies for reducing the overall energy consumption are a hot research topic within the ubiquitous computing (Chetty et al. (2008); Costanza et al. (2012)), HCI (Froehlich et al. (2010); Pierce and Paulos (2012); Jönsson et al. (2010); Gustafsson and Gyllenswrd (2005)) and environmental psychology (Siero et al. (1996); Carrico and Riemer (2011); Daamen, Dancker D. L. et al. (2001)) research fields. To date, most of the research has focused on saving energy at homes (Chetty et al. (2008); Costanza et al. (2012); Fischer (2008)), where motivational approaches to increase awareness are focused on a personal and financial level. Nevertheless, still few procedures have targeted public sector or common areas where people coexist, nor have they applied collective awareness approaches to tackle this major concern. The workplace is a very relevant case of study since our society spends at work roughly a half of the day, and this sector is now responsible for the $10 \%$ of the overall energy demand. A dramatic example is the United States of America where commercial buildings account for $36 \%$ of all U.S.A. electricity consumption as was stated in Energy Efficiency and Renewable Energy (2008). 
According to Carbon (2013), the energy consumed by work-appliances of collective use in offices, e.g. beamer projectors, coffee-makers, printers, screens, portable fans, kettles, and so on. represents more than $15 \%$ of the total and it is expected to rise above $20 \%$ in 2020 . This percentage could be reduced if workers increased their ecoawareness (Crosbie and Houghton (2012)). However, workers barely understand device's power consumption details and they are generally unconcerned about energy expenditure in these semi-public spaces.

This lack of understanding and awareness -both in sustainability and economic terms- might be attributed to the intangible nature of electricity, i.e. neither do they get any feedback from the appliances about their energy consumed nor are they provided with guidelines about how to use them efficiently (Chetty et al. (2008)).

The reviewed literature related to energy-awareness reveals that there are two trends to address the energy waste challenge.

1. complete technological approach where people is not really involved in the intervention (e.g. device's auto power down). An example of this approach is environment automation, where smart machines are energy-efficient and make the sustainability-oriented decisions on their own (e.g. Starik and Marcus (2000); Bush et al. (2009)).

2. human behavioural change approach where people bear the full responsibility of their decisions (e.g. switch off manually the devices when they are not in use) - Thieme, A. et al. (2012); Broms et al. (2010); Foster et al. (2012) -.

Our proposal is placed in the middleground of both trends, i.e. a mixed approach. It puts both eco-aware everyday things (in this study, a capsule-based coffeemaker which is an appliance of shared use) and workers on the same team to overcome the lack of energy consumption awareness. Our goal is to empower staff to act in a more energy-efficient manner than simply turning off the devices, leveraging the real-time eco-assistance provided by the eco-devices.

To this end, we have augmented a set of coffeemakers to make them perform two tasks: 1) report their daily usage pattern to a Cloud-server; and 2) get their usage prediction back in order to persuasively interact throughout the following week in a timely fashion with end-users.

The rationale of the former task is that a Cloud service will predict the appliances' next-week usage applying autoregressive ARIMA models. In other words, the Cloud-service infers when it would be advisable (in energy terms) that the coffee-makers remain turned on or off as a function of the number of people that previously used them, i.e. on the consumption patterns from past weeks. The use of ARIMA as predictive model is justified in the presented study. We have found that ARIMA models performed better than three different configurations of Artificial Neural Networks (ANNs) when predicting the time series of the number of coffees intakes. The latter appliances' task copes with providing persuasive feedback to users about the necessity of keeping the appliances on or in contrast, switch them off over certain periods of time throughout the work-day.

To test the effectiveness of our approach, three capsule based coffee machines placed in three different research laboratories were augmented. Each of them showed a different means of eco-feedback to the group, but only one of them featured the distinctive combination of a persuasive eco-aware everyday thing (mixed approach). The three group studies lasted one month with more than twenty people in total using the devices. The workers' experience was recorded through interviews made before and after the intervention, while the energy consumption details of the coffee machines' usage were as well logged during the whole trial.

The work is constructed over the basis of a work-inprogress (Casado-Mansilla et al. (2014)) and the communication and computing infrastructure presented in (Ventura et al. (2014)). In the former authors' reference, the emphasis was put over the design interaction of the coffee makers, while in the latter it was presented the whole architecture which supports the approach. This article makes a compendium of them with the novelty of: 1) confronting ARIMA models and ANNs to test and discussing their suitability and performance for the presented solution; and 2) extending the discussion and rationale of the eco-aware everyday things.

\section{Background}

Historically, sustainability-related scientific articles addressed motivational strategies to enhance sustainable behaviours in home environments (Chetty et al. (2008); Costanza et al. (2012); Froehlich et al. (2010)). Unfortunately, their findings and outcomes have been rarely tested in spaces where tens of people share appliances and electrical devices ( Foster et al. (2012)). However, since DiSalvo et al. (2010), Pierce and Paulos (2012), Brynjarsdóttir et al. (2012), and other researchers started discussing about the need to move beyond the individual level awareness towards larger-scales -like communities or groups- more research is being done in that field. The case of the workplace is not an exception. As Froehlich et al. (2010) suggested, in this field there 
is much to learn from the households' experiences and from previous literature in environmental psychology ( Siero et al. (1996); Carrico and Riemer (2011); Daamen, Dancker D. L. et al. (2001)).

In work environments, the feedback is usually not personalised but aimed to reach large groups. Furthermore, unlike what happens at their homes, workers do not receive any reward or financial incentive (one of the most powerful motivators) when they save energy at work. Considering these premises, we argue that it is challenging to switch per se the common sustainability intervention techniques from homes in the workplace Yun et al. (2013a). For the sake of a better understanding, we grouped some of the reviewed energyawareness studies in the workplace by the type of strategy they followed. Table 1 shows a summary of all referenced works.

Energy saving driven by behaviour change Siero et al. (1996) introduced the concept of comparative energy consumption feedback in organisations. They demonstrated that such feedback is more useful when it is reported with other data to compete with (e.g. the energy saving of other group). Carrico and Riemer (2011) developed two interventions in the workplace to evaluate the effectiveness of group-level feedback versus peer education. Their results revealed that the former methodology exhibits a better potential for reducing energy use than the latter. Schwartz et al. (2010) investigated the role of workers to save energy. They collected energy data from a group within a large organisation to conduct a series of participatory action research to spark reflection and discussions about energy wastage. Similarly, Foster et al. (2012) described how a qualitative research methodology, i.e. workshops in the public sector analysed by applying grounded theory, can disclose the perception that workers had about their energy use and how to enhance it through technology-led feedback.

\section{Energy saving driven by technology Based on}

Schwartz et al. (2010), Jahn et al. (2011) created a Ubicomp system to support energy-awareness in work environments. Similarly, Industrial environments have been studied by Jönsson et al. (2010) in an exploratory research where modifications in tangible design and electricity visualisation of light-torches affected the ecobehaviour in the workplace. Yun et al. (2013a) conducted a literature review, based on Yun et al. (2013a), to investigate the appropriate persuasive methods to be applied on building control systems to reduce the unsustainable energy practices. To this end, they developed dashboard-controllers that enable office workers to control their energy use. Finally, in the commercial sector there are many devices that help to save energy ${ }^{123}$. However, they do not feature adaptive behaviours which can be timely settled in the users preferences or comfort. The Nest thermostat is the closest approach to our eco-aware everyday things concept, over all its model conceived for business spaces ${ }^{4}$. Its Auto-Schedule mode learns when people go in and out of the office, Nest's Auto-Away will save energy while everyone's gone and Nest's Thermostat Lock will keep co-workers from changing the temperature too much. To the best of our knowledge, there has not been any research carried out which has used Nest Thermostat in offices. However, there are interesting findings from its experience in households (Yang and Newman (2013)). In that article, the authors highlighted the relevance of using machine learning techniques and sensing technology to make the eco-feedback more successful.

The majority of the reviewed work used group monitoring and informative feedback as the only tools to motivate energy awareness. Indeed, it has been largely demonstrated that both techniques have a great potential to save energy (Winett et al. (1979); Darby (2006); Fischer (2008)). The informative feedback was presented in different ways in these studies: Comparatively by Siero et al. (1996), through weekly e-mails of the group performance by Carrico and Riemer (2011), associated to everyday activities (Schwartz et al. (2010); Jahn et al. (2011)), breaking down by device-type through a webbased application (Yun et al. (2013a)) or with feedback built-in in the own appliance as Yang and Newman (2013) performed.

We consider that the previous feedback strategies are not enough to bring about efficient use of electrical appliances in common shared spaces, such as the workplace. Thus, users provided with only informative eco-feedback are not able to know in real time whether the action that they are performing is "environmentally adequate" or not. Furthermore, it is unlikely to expect long-term energy-efficient practices if people in working environments are supposed to act individually and bear the full responsibility of their energy related actions. This work emphasises the need to assist or facilitate the real-time decision-making process necessary to reduce energy consumption on everyday activities at

\footnotetext{
1 http://www.alertme.co.uk

2 http://www.currentcost.com

3 http://www.theowl.com

4 https://nest.com/blog/2012/08/23/nest-thermostatsfor-business /
} 


\begin{tabular}{|c|c|c|c|c|}
\hline \multicolumn{5}{|c|}{ Background Summary } \\
\hline Study & Strategy & Technology & Type of workplace & Evaluation \\
\hline Siero et al. (1996) & $\begin{array}{l}\text { Comparative feedback } \\
\text { on energy consumption }\end{array}$ & Weekly graphic displays & Metallurgical company & $\begin{array}{l}\text { Goal-setting; } \\
\text { questionnaires }\end{array}$ \\
\hline Carrico and Riemer (2011) & $\begin{array}{l}\text { Group-level feedback vs. } \\
\text { Peer education }\end{array}$ & $\begin{array}{l}\text { Monthly e-mails with } \\
\text { graphic of Kwh consumed }\end{array}$ & University campus & Goal-setting \\
\hline Schwartz et al. (2010) & Participatory action research & $\begin{array}{c}\text { Smart metering; } \\
\text { graphic of kWh consumed }\end{array}$ & Institute for applied research & $\begin{array}{l}\text { Reflection workshops; } \\
\text { online-questionnaires }\end{array}$ \\
\hline Foster et al. (2012) & $\begin{array}{c}\text { Workshops and } \\
\text { energy related task designing }\end{array}$ & 管 & $\begin{array}{l}\text { Five universities and } \\
\text { Industry }\end{array}$ & Grounded theory \\
\hline EnergyPulse Jahn et al. (2011) & $\begin{array}{c}\text { Workshops and design } \\
\text { support systems for saving energy }\end{array}$ & $\begin{array}{l}\text { Sensors: power consumption; } \\
\text { presence and user interactions }\end{array}$ & Institute of applied research & Business Ethnography \\
\hline Watt-Lite Jönsson et al. (2010) & $\begin{array}{c}\text { Objects as mediators; } \\
\text { comparative energy consumption }\end{array}$ & $\begin{array}{l}\text { Web-site; light projected from torches } \\
\text { related with factory's e-consumption }\end{array}$ & Eight industries & $\begin{array}{l}\text { Questionnaires; notes; } \\
\text { photographs }\end{array}$ \\
\hline Yun et al. (2013b) & $\begin{array}{l}\text { Self-monitoring; advice; } \\
\text { comparison; controls }\end{array}$ & Dashboard for Occupants & $\begin{array}{c}\text { University and } \\
\text { government research lab }\end{array}$ & $\begin{array}{l}\text { Questionnaires; } \\
\text { energy saved }\end{array}$ \\
\hline $\begin{array}{l}\text { Yang and Newman (2013) } \\
\text { (NEST) }\end{array}$ & $\begin{array}{l}\text { A green leaf rewards } \\
\text { for efficient usage }\end{array}$ & $\begin{array}{l}\text { Machine learning: Auto-Schedule } \\
\text { Auto-Away; Thermostat Lock }\end{array}$ & $\begin{array}{l}\text { (Not tested in workplace) } \\
\text { Nineteen households }\end{array}$ & Interviews \\
\hline
\end{tabular}

Table 1 Summary of academic studies related with energy-awareness in working environments grouped by the type of strategy designed, eco-feedback technology used, method of evaluation applied and the location where the study was carried out.

work, since it is a promising approach to reduce consumption (Fischer (2008); Yang and Newman (2013)) and none of the previous studies addressed it.

\subsection{Motivational Strategies to Increase Awareness}

Eco-aware everyday things take advantage of the knowledge acquired for timely interaction with concerned users. They are able to encourage users to perform energyefficient actions such as keeping appliances on, or in contrast, switch them off over certain periods of time during the work-day. The theoretical strategies that support its design were introduced in a work-in-progress (Casado-Mansilla et al. (2014)). They are reviewed in depth hereafter.

\subsubsection{Persuasive Interaction}

Our society is increasingly influenced by everyday technological products. Everyday device designs now have the potential to change our opinions and behaviours through them ('Objects as mediators', see Chapman (2005)). Persuasion methodologies towards a sustainable lifestyle or health care have been largely covered in the Psychology literature. However, it was only in 2003 when Fogg (2003) published a book where persuasion and technology were linked, i.e. computers or persuasive technology as main actors towards changing people's attitudes or behaviours. Since then, most of the research works on sustainable HCI have based their theoretical rationale on this model (DiSalvo et al. (2010)). Eco-aware everyday things are also aligned with Fogg's theories: $a$ ) a technology-led approach; $b$ ) it follows the Fogg Behaviour Model -FBM- (Fogg (2009)): 1) increasing people's motivation with eco-feedback; 2) easing the decision-making; and 3) triggering suggestions in the right moment, i.e. when people is about to operate the appliances; c) finally, they feature some of the persuasive techniques described on his framework, e.g. authority, reduction or suggestion.

\subsubsection{Ambient Eco-feedback}

DiSalvo et al. (2010) discussed the mismatch between pervasive technology and ambient awareness stating that "many persuasive systems are ambient, based on the idea that the ambiently provided information will persuade the user to behave sustainably". Eco-aware everyday things are devised upon the ambient awareness premise mainly to be unobtrusive and to present the suggestions subtlety as proposed by Schmidt et al. (1999). There are some successful examples of ambient ecofeedback built-in within the objects in the literature that helped us to define our proposal (Arroyo et al. (2005); Broms et al. (2010); Gustafsson and Gyllenswrd (2005); Jönsson et al. (2010)).

\subsubsection{Teammates}

Eco-aware everyday things raise energy awareness by making visible to humans their own energy waste with ambient media. However, these 'things' are conceived to be more than mere energy consumption reporters. They are designed to be eco-activists devices, i.e. everyday things which actively aid people to take more sustainable decisions in common areas in order to save energy. We are not the first to consider that humans and technology can and should work together, i.e. to be allies. Nass et al. (1996) already investigated about team affiliation between humans and computers. They concluded that interdependence cues were pivotal to create a team relationship. Indeed, the subjects under interdependence conditions were found to be more cooperative and open to influence from the computer. Our work constructs over the Nass' findings applying their research methodology to the field of smart everyday things. 


\subsubsection{Social Networks}

Internet-connected objects may contribute to reduce energy consumption by giving them an 'active-voice' towards energy efficiency. Social Networks are currently a promising channel to pave the way between human beings and these intelligent objects for sustainability purposes (Thieme, A. et al. (2012); Foster et al. (2010)). Moreover, these networks are now experiencing an exponential growth, e.g. Twitter has officially become the next big thing in terms of Internet social phenomena gaining worldwide popularity, with over 400 million active users as disclosed by Farber (2012). Therefore, if we now want to reach thousands of users to raise their energy awareness (Brynjarsdóttir et al. (2012); Pierce and Paulos (2012); DiSalvo et al. (2010)), a simple 'tweet' could achieve the task. Eco-aware everyday things are provided of a public Twitter profile where the breakdown of their daily energy consumption is reported.

\section{Eco-aware Everyday Things}

The eco-aware everyday things have been devised to increase the awareness about the careless waste of energy resources in common areas when using devices of shared use, e.g. printers, coffee-makers, kettles, beamers and so forth. One typical inefficient end user behaviour is to leave these devices on when nobody is using them. Absent mindedness and comfort are the typical causes of that issue as was suggested by Yang and Newman (2013). Most of the reviewed literature on the field addresses this previous scenario. However, energy conservation is not only about switching off devices after using them, but also about empowering people to operate them in an intelligent manner to minimise energy wastage. Eco-aware everyday things should also be devised to reduce the ecological impact that would cause the replacement of every old-fashioned consumer appliance by new eco-friendly Internet-connected ones. Therefore, any everyday appliance could be transformed into eco-aware by simply embedding a specific purpose electronic platform within it. We call this platform, eco-adaptor. Eco-adaptors provide collective appliances with insights about the optimal operational mode in each case. Applying machine learning techniques with their real usage patterns, devices know when they should switch from an operation mode to another during the day in order to save the most of energy.

\subsection{Why do we need them?}

Worldwide campaigns to raise awareness of the consequences resulting from the waste of resources have succeeded in establishing social norms in favour of recycling. Consumers are generally positive when something can be recycled. However, these initiatives have forgotten the other two R's of Reduce-Reuse-Recycle (EUComission (2008)). Reusing something already created saves more energy than recycling it. However, the key to reduce the ecological footprint lies in the reduction principle. Eco-aware everyday things intend to address these two issues. First, they help to reduce the energy consumption whilst in operation, as well as they help to reduce electronic waste since they would end in the landfill if the whole society decides to replace their oldfashioned appliances by new smart ones. Second, less frequent use of their components increase their durability and potential reuse. Moreover, eco-aware everyday things not only decide what the optimal behaviour to meet these goals is, they also collaborate with their users by providing appropriate feedback. Therefore, users can even learn to reduce energy waste on other non ecoinstrumented devices.

The following scenario illustrates situations in which an eco-aware everyday thing could help reducing power consumption and suggesting users what is the appropriate action in each case.

Elisa wakes up in her apartment. After a shower, she uses her new capsule-based coffee-maker to prepare a cup of coffee. After breakfast, she turns off the lights in the kitchen, but forgets to turn the coffee-maker off! (one decision she will regret later). Elisa leaves home and goes to work. After arriving to her company, she goes to the WC. Despite the clarity that comes through the windows of the toilet, the lights were on. She does not think in turning the lights off when leaving. By noon, Elisa visits the shared lounge to prepare a cup of coffee. She turns the coffee-maker on, puts a capsule of her favourite flavour and waits. When she finishes, she doubts whether to turn the coffee-maker off or let it on (standby mode), because at that time there are many colleagues who also have a coffee break.

As we can see, there are several types of devices in this scenario: 1) personal-use devices whose power consumption is evident (kitchen lights); 2) personal devices whose power consumption is less evident (home coffeemaker); 3) shared devices whose power consumption is evident (toilets lights); and 4) shared devices whose power consumption is less evident (shared coffee-maker in the office). Very few people forget to switch off the lights at home. Likewise, it is very unlikely to leave a 
hair dryer on, while it is usual to forget to turn off a coffee-maker or an electric heater (although their power consumption is similar). Indeed, awareness about the energy consumption of collective appliances is negligible when compared with our own personal appliances as pointed by Schwartz et al. (2010). In these collective cases it would be useful to provide users with some help. Regarding the usage of shared devices, another difficulty occurs: the diffusion of responsibility. Darley and Latane (1968) studied this phenomenon in relation to human behaviour in emergencies. They concluded that the process that leads people to act is not a simple decision, but occurs within a decision tree. People have to notice, interpret, take responsibility, learn the proper way to act and finally act. Doubts in any of these steps will make difficult the final action. For this reason, an eco-aware everyday thing should help Elisa to realise that there is no need to leave the toilet lights on, or recommend to her that in some parts of the day is more efficient to leave the coffee-maker in standby. In a nutshell, it might foster her to take responsibility, know the proper way to act and finally decide to act towards reducing energy consumption effortlessly.

\section{Study Design}

The work presented aims to study whether the design of the eco-aware everyday things and the underlying infrastructure that support them is adequate to: 1) combine soft computing techniques with persuasive ecofeedback to cope with energy inefficiency; 2) increase people's energy awareness making this device appears as an energy authority. Fogg (2003) found that people who perceive technology as an authority are more susceptible to persuasion by that technology.; 3) create a sense of interdependence between people and the eco-augmented appliances in order to ally people and machines to develop a team relationship towards energy saving and 4) to test and evaluate the effectiveness of the eco-aware everyday things' design with regard to other designs based on informative eco-feedback.

To this end, we performed a one month betweenthree-groups study at a workplace. The study was related with the usage of an appliance of common use, a capsule based coffee-maker. The three capsule-based coffee machines were augmented with technology. They were all able to collect and log their energy data and to provide eco-feedback to the corresponding users. Nevertheless, only one of them featured the full distinction of an eco-aware everyday thing.

\subsection{Participants and Field of Application}

The participants that took part in the study were 20 members belonging to three different laboratories. The laboratories are placed within a large technological institute that is attached to the authors' University. The population was therefore selected by convenience sampling. Although the laboratories belong to the same institute, each had its own working-room. From now on, we call these laboratories by their name for the sake of clarity throughout the study description: S3Lab, ProtoLab and SmartLab. The 20 participants belonged to these laboratories: 8 people, 4 people and 8 people, respectively. The participants' age ranged between 20 and 40 years, they were all males and engineers in telecommunications or computer scientists. Among the participants, 2 were lecturers, 4 post-doctoral researchers, and the remaining $14, \mathrm{PhD}$ students. All the participants used to have coffee daily in a range of one to three coffees per day. The three laboratories had a loungecorner at the back of their working-room where the coffee-maker was located.

\subsection{Apparatus and Materials}

To deploy the eco-aware everyday things at geographical scale, necessary infrastructure must be in place for their operation. To this aim, the authors devised an infrastructure called ARIIMA in Ventura et al. (2014). ARIIMA uses Autoregressive Integrated Moving Average (ARIMA) predictive model to forecast devices' nextweek usage (i.e. a time-series of the number of coffee intakes).

The ARIMA model, also known as the Box-Jenkins model, is widely regarded as the most efficient forecasting technique in Social Sciences and is used extensively for time series. The use of ARIMA for forecasting time series is essential with uncertainty because it does not assume knowledge of any underlying model or relationships as in some other methods (Ariyo Adebiyi et al. (2014)). ARIMA assumes that past patterns will similarly occur in the future, and therefore are predictable (Box and Jenkins (1990)). These soft computing techniques applied to the operation of a shared coffee-maker to discover its optimum energy-efficient mode were covered in a previous work of the authors López-de Armentia et al. (2014). In that work, we distinguished the two typical operation modes of appliances and we disclosed their associated power consumption: 1) On-Off mode, consisting in repetition of actions "switch on", "waiting for the coffee machine to heat", "prepare the coffee" and immediately "switch off"; and textit2) Standby mode, 
in which the appliance is permanently ready to be used and no long warming time is needed when one wants to prepare a coffee. One of the goals of López-de Armentia et al. (2014) was to theoretically demonstrate the convenience of introducing a new operating mode in appliances to save energy depending on the number of coffees per hour. Thus, a mode that adjusts the coffee machine's operation depending of the usage it is subjected to. In rush hours (3 coffees or more per hour) the coffee-maker should remain on (Standby mode), while during periods of lower use it has to be switched off (On-off mode).

In this work we strengthen our decision to use ARIMA models based on three premises: 1) they are relatively more robust and efficient than more complex structural models in relation to short-run forecasting (Khashei and Bijari (2010)) as is our case; 2) they only require data on the time series in question; and 3) for our specific time series, the chosen ARIMA model (see Lópezde Armentia et al. (2014) for the model identification) outperforms three configurations of Artificial Neural Networks (ANNs) as is demonstrated in the Apendix section.

\subsubsection{ARIIMA architecture}

As stated before, the eco-aware everyday objects have an underlying RESTful Cloud-based architecture which supports their operation. The aim of this architecture is: 1) to alleviate the computational process of the electronic platform (eco-adaptor) that is attached to each device. 2) to manage each of the eco-aware appliances within the ecosystem in order to receive appropriately the device's energy data. In the frontend, eco-adaptors tailor the saving schedules for the referred devices. The eco-adaptors consists of:

- iBoard Pro, which is an Arduino MEGA compatible microcontroller featuring an Ethernet interface, uSD card slot and RTC clock (The red board that appears in of Figures 1 and 2)

- Split core current transformer, also called 'non-invasive current sensor' to measure the energy data.

In the backend, the RESTful main server provides a set of REST APIs to receive energy data from appliances and to generate the weekly forecast associated to each sustainable device in order to give them back such information.

The data storing of energy consumption events can be observed in Figure 1. Whenever a new energy event is detected, the eco-adaptor captures some useful information like its timestamp (through a battery-powered

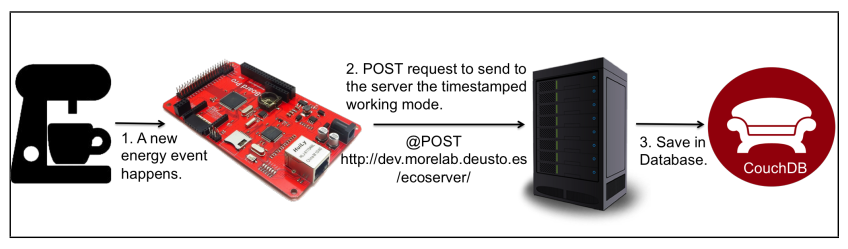

Fig. 1 Detection of a new energy event and storing of useful data into the RESTful Cloud-based server.

RTC clock which is daily synchronised with a pool of NTP servers), the energy value consumed in Wh, the state in which the machine is set (standby, working or warming), and so forth. Then, it executes an HTTP POST request against the RESTful server. The request issues a JSON object which contains the information retrieved. The server stores the JSON object as a document inside a CouchDB NoSQL database.

The energy dataset stored in the database is used by ARIIMA architecture to predict the appliance's operating mode for the working days of the next-week (from Monday to Friday). The computational phase is done weekly (on Sundays). The process is described in Figure 2 .

Following the schema, it is observed that the first operation performed is to search for the number of coffees consumed in each of the hour slots along the previous 23 working days. The information returned by the CouchDB database is curated by the server in a dataset processable by an R script (R, R-Core Team (2013)). To perform this data transformation, every working day is divided into slots of 12 hours (from $7 \mathrm{am}$ to $7 \mathrm{pm}$ ) and the total amount of coffees made in each hour is calculated. Using this vector as input parameter, the forecasting is executed. Using the output vector of 60 values, it is logically compared whether each of the values exceeds or not the threshold of three coffees (this threshold was calculated in a previous work from the authors López-de Armentia et al. (2014)). The aim is to decide whether the coffee-maker should remain on (Standby mode), or in contrast switch it off (On-off mode) if the number of coffees predicted is lower than three. On the former case, the prediction time slot is set to "1" while the latter case is set to " 0 ". This binary vector (i.e. 60 bits of binary data grouped in 12 bits for each day) is saved as JSON object inside the database (See Listing 1). 


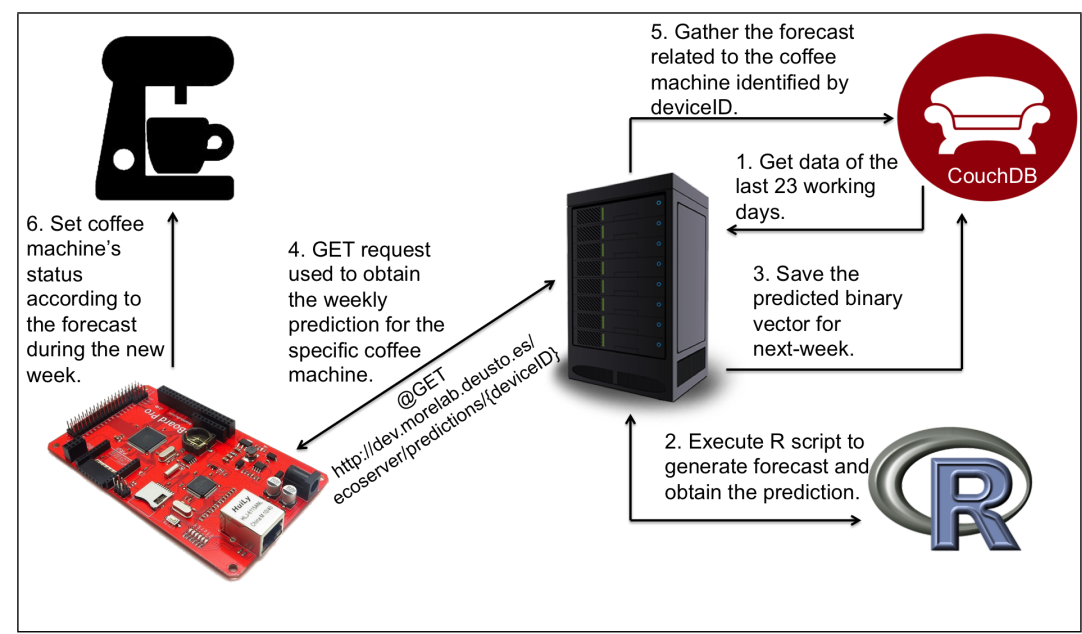

Fig. 2 Computational phase to predict the next-week usage and transmission of forecasted values to the eco-aware smart thing.

Listing 1 Predicted 60 bits of binary data. Each bunch of 12 bit corresponds to each working-day (7am-7pm) of the new week. \{

"deviceID": "Lab1",

"prediction": "0011100001100111000011..."

Whenever an eco-aware coffee-maker has to update its weekly operation schedule, it has to perform an HTTP GET request against the Cloud-Server sending its deviceID. When the server receives the request, it queries the database (filtering the query by deviceID) and it obtains a JSON object containing the predicted binary data. The server sends this vector back to the microcontroller which saves it in its EEPROM memory. In this way, every day the eco-adaptor reads 12 bits from the EEPROM which lets it inform its users about the most-efficient way to operate the appliance in the typical usage time slots.

\subsection{Procedure}

Before starting the study, we recorded the usage of the SmarLab's shared coffee machine. We found that its usage was pretty random. That is, people applied different operating modes when preparing a hot-drink: some left the coffee-maker on, i.e. Standby mode, and others turned the device off, i.e. On-Off mode after its utilisation. Such randomness, which could be extrapolated to any other electrical appliance of collective use, was noticeably correlated with high rates of energy waste. Therefore, we conducted a one month field-study to obtain deeper insights about how to encourage energy ef- ficient devices' operation to save energy. The study was divided in three phases.

\subsubsection{Phase 1: A pre-trial survey}

In the first round, two researchers ran personal interviews. We collected personal information from participants, i.e. age, genre, laboratory, coffee habits, and so forth; all participants signed an agreement to allow the collection of energy data from the coffee-maker throughout the corresponding month (for ethical reasons, in this article their names are changed to fake ones). However, the purpose of the study was hidden to the subjects to lower the intervention bias; we asked participants about energy metering and efficiency; we asked them some simple questions on sustainable practices in private contexts and in public ones; finally, we tried to get their insights about solutions to overcome energy leakages and their preferred motivational strategies to increase people's awareness. The purpose of retrieving this information was to have a general understanding about the knowledge that participants had on energy related concepts and their perception of energy consumption at work. This information was coded as an attribute to categorise participants according to their energy-related background.

\subsubsection{Phase 2: The development of the field study}

In this phase, the energy data of the three appliances was logged. Along the 4 weeks of intervention, the means by which each of the coffee-makers presented their energy consumption to users, i.e. eco-feedback, was changing weekly (The Table 3 summarises the pilot). The $1^{\text {st }}$ week, the three coffee machines were left without 


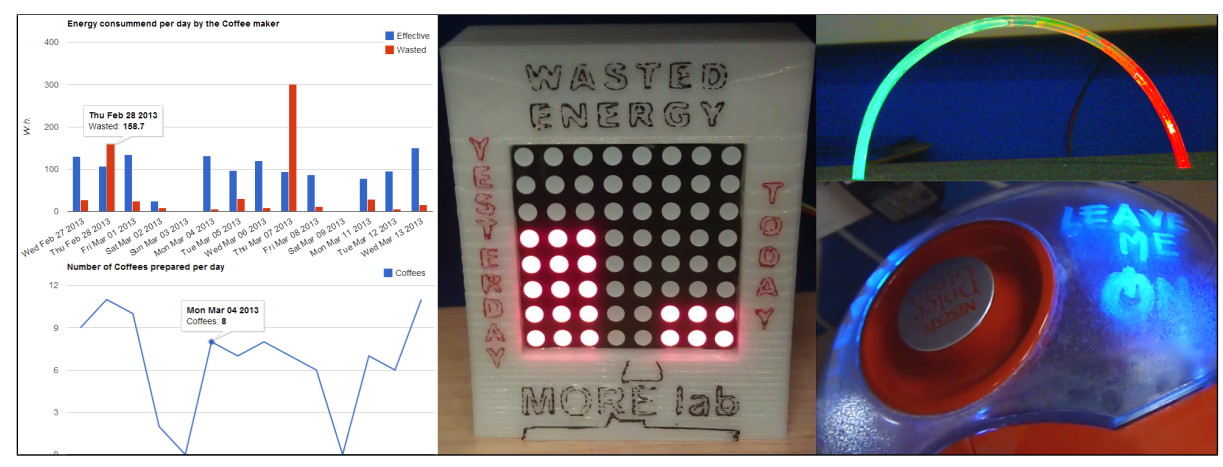

Fig. 3 a) Dashboard showing the wasted and effective energy in S3Lab; b) The physical display allows to compare the current wasted energy with the previous day; c) The light arch shows the increase of the wasted energy, while the coffee-maker prevent people with subtle advices about how to behave efficiently.

changes, i.e. we did not apply any intervention beyond collecting data. This week was considered as baseline. The $2^{\text {nd }}$ week, a public Twitter profile was created for each coffee-maker: @S3LabCoffee, @ProtoLabCoffee and @SmartLabCoffee. In that way, the workers were able to follow their performance by seeing their daily published energy consumption in wh on Twitter. All the subjects implied in the study were notified about this new feature. Twitter was selected as a communication channel, because according to the interviewees in phase 1, it was their most used social network, $68 \%$ of the subjects indicated to use Twitter daily, and in general there was a general agreement that it is easy to reach all the users of a laboratory with just one 'tweet'. In their accounts, the social appliances published daily the number of coffees prepared and their associated energy consumption. The expected goal was that workers become followers of the appliance's Twitter profile in order to keep updated of its performance. So, followers may check if they have reduced or not their energy wasted with respect to previous days. This kind of social eco-feedback allows to keep track the device's consumption in an effortless manner.

Before starting the $3^{\text {rd }}$ week, each of the each of the socially-enabled devices was augmented with different eco-feedback technologies. These new features remained until the end of the intervention, i.e. the whole $3^{r d}$ and $4^{t h}$ weeks. The Figure 3 presents the different eco-feedback methodologies which were leveraged in the the three coffee machines.

$\boldsymbol{S} \mathbf{3 L} \boldsymbol{a b}$. The left side of the Figure, a), shows a web interface which was developed for the participants of the S3Lab where coffee's intakes and energy wasted were depicted. We placed on the top of their coffee machine a picture of a QR-code which linked to the website where this informative eco-feedback was given. The rationale of the QR-code was to remember and ease the process of accessing to the website. Dashboards are one of the most typical informative and self-monitoring eco-feedback means (Yun et al. (2013b); Costanza et al. (2012); Schwartz et al. (2010)).

ProtoLab. In ProtoLab, we placed a 3D physical display close to the coffee-maker that mimics a side-by-side bar chart (it can be observed in the middle of Figure 3, b) ). This kind of informative eco-feedback showed to users their previous day's wasted energy (the longest red bar of the led matrix - left side -), whilst the energy being wasted on the current day was shown on the right side of the physical display. This design was inspired by the Energy Aware Clock of Broms et al. (2010). The rationale of this design is based upon the challenge of self-perfomance. Thus, people from ProtoLab were expected to try to not overtake the left-side bar chart.

SmartLab. The eco-adaptor and its associated interaction for increase energy-awareness (pictures in the right side of Figure 3,c), were installed in the coffeemaker of SmartLab laboratory. The usage pattern of the previous two weeks of the study was reported to the Cloud-server to get-back its efficient-operation schedule. Before the beginning of the $3^{r d}$ week, we explained to the eight SmartLab members that the coffee-maker was able to learn how people use the appliance, but no more because we wanted to get after insights about the interaction comprehension. The design of the ecoaware coffee maker was based on the eco-aware everyday thing's rationale presented the Background Section. Table 2 provides a summary of it and the background for each of the device's features.

The eco-aware coffee-maker provided two types of ambient feedback to its users. 1) Informative visual feedback through an ambient light arch; and 2) subtle suggestions about how to operate the device ap- 


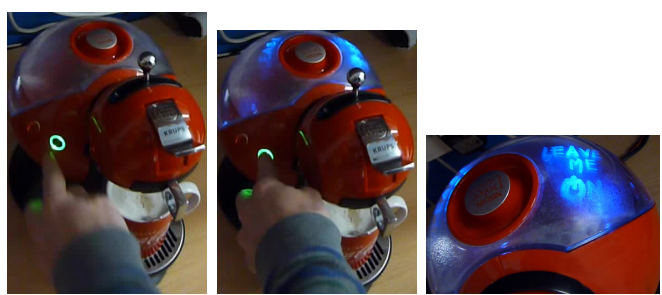

Fig. 4 On the first image a user approaches with their finger to switch off the device. In the second and third picture, it is shown how the device informs to the intended user to not do it by activating a set of ultraviolet lights LEDs that were embedded underneath the water tank.

propriately through built-in markers in the machine itself. 1) The ambient light arch, which was placed close to the coffee-maker - right-top of Figure 3, provides information about the energy that is being wasted each day. The arch starts the day being completely green, but it progressively turns into red as the wasted energy increases. 2) The subtle suggestions are triggered when the eco-aware coffee-maker's schedule informs that throughout a whole hour-slot it is more appropriate to remain in standby mode rather to switch itself off. The coffee-maker can detect when somebody is about to switch the device off after a coffee preparation - a proximity sensor pointing to the start-button has been attached to detect such action. When such detection occurs, the eco-aware appliance suggests the corresponding user to avoid it depicting the message: 'Please, leave me on.' This message was before disguised in the outside of the water container and can only be seen under ultraviolet light. The process can be appreciated in the snapshots of Figure 4.

\subsubsection{Phase 3: A post-trial questionnaire}

In the second round of personal interviews, at the end of phase 2, we wanted to get the participant's insights about the proposed interactions, their comprehensibility, understanding and usefulness, the relevance that they gave to the intervention towards reducing energy and the motivations that have been issued to save energy during the treatments. The questionnaire was divided in two parts and was conducted by two researchers to strengthen the studio reliability: 1) A semi-structured interview; 2) A self-report of eighteen items in a 5point Likert scale. The self-report was structured in four blocks to record the participant's insights about their affiliation and interdependence with the coffeemachine, the role of authority that they attributed to the device, and finally three items devoted to extract the participant's acceptance around the inclusion of the smart-device in their work routine. Most of the items were based on the scale used by Nass et al. $(1996)^{5}$. Assuming that there were certain similarities among the three groups (similar number of people, socio-economic profile, participants background and schedules of coffee intakes), Pearson chi-square analysis was used to assess the statistical significance between the answers of the group that interacted with the eco-aware coffee-maker and the groups which did not.

\section{Results and Analysis}

The pre-trial survey confirmed that interviewees were not concerned with energy expenditure in their workplace and that they lacked of motivation to reduce it. Indeed, four in five felt confident when saying that the use that they do when using the appliances in the workplace is energetically insignificant. $74 \%$ asserted to be much more aware about energy consumption in their homes than in their workplace. The remaining $26 \%$ said to be equally concerned in both spaces. Most of them attributed financial causes to their replies.

For example user A stated: "That [the energy waste issue at work] does not directly affect to me. It is a sharing thing and in public spaces I lose track of responsibility" or user B argued: "I am not motivated to reduce energy since the action of one guy is diluted if the group does not take joint actions to tackle it"

We found a general agreement from respondents about the devices that consumed the most of energy in their workplaces: lights, PCs, screens and shared appliances. When asked about the general policies that they would put in practise to reduce consumption, $47 \%$ proposed technologically-led, e.g. e-meters, automation, sensors, etc. 31\% human-centred approaches (switch by hand, reflection by example) and the rest a mixed approach, e.g. fair competitions and gamification. At the end of the survey we discussed with the interviewees about energy concepts, e.g. energy waste, efficiency, standby, and so on, and about technologies that could help to reduce consumption. There was a general agreement that in case of knowing more about energy waste in real time, their perception of energy waste would increase. Moreover, they asserted that the more informed they were, the better their actions towards energy efficiency would be.

\subsection{Quantitative Data}

The quantitative data can supporting evidence on the effectiveness of each methodology when saving energy

5 The whole questionnaire in its Spanish version is available on http://tiny.cc/xrasbx 


\begin{tabular}{|l|c|c|}
\hline \multicolumn{1}{|c|}{ Feature } & Rationale & \multicolumn{1}{c|}{ Bco-aware Coffee-maker Design } \\
\hline Intelligence & $\begin{array}{c}\text { To infer the moment when the coffee-maker } \\
\text { is likely to be used. }\end{array}$ & $\begin{array}{l}\text { Box and Jenkins (1990) assumed that past patterns will similarly occur } \\
\text { in the future, and therefore are predictable. }\end{array}$ \\
\hline Persuasiveness & $\begin{array}{c}\text { It interacts persuasively with users to raise } \\
\text { their energy-awareness. }\end{array}$ & FBM Fogg (2009): Motivation, simplicity and triggers. \\
\hline Ambient awareness & Ambient light arch + Hidden messages. & $\begin{array}{l}\text { Not relying in behaviour change elicited by direct requests, } \\
\text { be unobtrusive and to present the suggestions subtlety Schmidt et al. (1999). }\end{array}$ \\
\hline Social presence & $\begin{array}{c}\text { Collective awareness with just a daily 'tweet'. } \\
\text { A promising channel to link human beings } \\
\text { and intelligent objects for sustainability purposes Thieme, A. et al. (2012). }\end{array}$ \\
\hline Team affiliation & $\begin{array}{c}\text { Assist users in their decision-making } \\
\text { when operating the coffee-maker. }\end{array}$ & $\begin{array}{l}\text { In Nass et al. (1996) the participants under interdependence conditions were } \\
\text { to be more cooperative and open to influence from the computer. }\end{array}$ \\
\hline
\end{tabular}

Table 2 The rationale followed to design the Eco-aware coffee-maker associated with the background which supports each feature.

at work. The total energy consumed by a capsule-based coffee-maker can be broken down in three different states depending on the device operation: (1) Warming a phase where one has to wait for the coffee machine to heat the water; (2) Standby the energy consumed whilst the device is on without being used - no long warming time is needed when one wants to prepare a coffee; (3) Working the device is preparing a hot drink. Warming and Standby are two states where the coffee-maker is leaking energy, so both are tagged as non-effective energy. The Working state, is tagged as effective energy. We use these energy proportions to compute a simple comparative unit of measure to evaluate the laboratories' performance, namely the cost of a cup of coffee in Wh. Such a cost is calculated weekly in terms of the laboratory members' efficiency when operating their coffee machine. The higher their awareness to not waste energy, the lower the cost in wh of a cup of coffee.

In Table 3 it can be appreciated that the appliance that performed the best at the end of the trial, $3^{r d}$ and $4^{\text {th }}$ week, it was the eco-aware coffee-maker placed in SmartLab laboratory. As it is observed, giving ecofeedback using Twitter has not lead to a significant change in the device usage (the $2^{\text {nd }}$ week the energy cost of a cup of coffee was even higher than the baseline). Nevertheless, transforming the coffee-maker into an eco-aware everyday thing the proportion of Noneffective energy consumption was considerably reduced. The cost of a cup of coffee also decreased: $38.12 \mathrm{Wh}$ the 1 st week and $49.19 \mathrm{Wh}$ the $2 \mathrm{nd}$ decreased to $34.82 \mathrm{Wh}$ the 3rd week and $35.52 \mathrm{Wh}$ the 4 th at the end of the study. Analysing the S3Lab data we found no cues of improvements in the device usage. Contrary to what was expected, the cost of a coffee performed uneven fluctuations throughout the whole study. The cost of the coffee cup surprisingly raised up to $78,61 \mathrm{Wh}$ in its maximum, which is the double of the baseline. These results could be attributed to the time period elapsed between the action of using the device and the feedback provided by the dashboard.

ProtoLab data were also examined. This laboratory presented the highest rates of non-effective energy due to the absent-mindedness of leaving the device uselessly on in three out of four weekends. Therefore, the cost of a cup of coffee was also the most expensive if we traduce from energy units to money. The introduction of Twitter in the $2^{\text {nd }}$ week and the physical display in the $3^{\text {rd }}$ did not lead to a significant change of device usage. Contradictory again, the energy cost presented unfavourable energy rates. However, the $4^{\text {th }}$ week was more efficient than previous ones. This radical change of tendency could be attributable to a better understanding of the interactive metaphor of the $3 \mathrm{D}$ graphic bar (middle picture of Figure 5). The users could have better understood that the more the energy they waste throughout the work-day, the quicker the right bar will increase, even overtaken the energy wasted the day before.

The energy data evidence that the eco-aware coffeemaker has been used more efficiently than the other two. The top of Figure 5 shows the breakdown of the proportion of non-effective energy consumption to the total in SmartLab on each of the four weeks. The dashed horizontal line represents the monthly-mean which is $42 \%$. The bottom of Figure 5 illustrates a side-by-side boxplot to analyse in depth a presumably increase of energy awareness of SmartLab members. The plot represents the spread of the non-effective energy wasted in the SmartLab along each of of the weeks that the trial lasted. The horizontal shaded line denotes the median of the month. It can be appreciated that the $3^{\text {rd }}$ and $4^{\text {th }}$ weeks the distribution of non-effective energy ratios changed radically, i.e. the energy wasted decreased and the data dispersion was concentrated near their median which means a more uniform usage of the appliance.

As the Table 3 shows, the data from the different laboratories is not uniform along the intervention, and the differences in Wh among them is more than relevant. Absent-mindedness, comfort, and getting used to the novelty of the appliances features are explanatory variables for the former statement, while for the latter, the diverse models of coffee-maker placed in each labo- 


\begin{tabular}{|c|c|c|c|c|c|c|c|}
\hline & \multirow{2}{*}{$\begin{array}{c}\text { 1st Week } \\
\text { BASELINE } \\
\text { coffee cost }(\mathrm{Wh})\end{array}$} & \multicolumn{2}{|c|}{ 2nd Week } & \multicolumn{2}{|l|}{ 3rd Week } & \multicolumn{2}{|c|}{ 4th Week } \\
\hline & & Eco-feedback & $\begin{array}{c}1 \text { coffee cost } \\
(\mathrm{Wh})\end{array}$ & Eco-feedback & $\begin{array}{c}1 \text { coffee cost } \\
(\mathrm{Wh})\end{array}$ & Eco-feedback & $\begin{array}{c}1 \text { coffee cost } \\
(\mathrm{Wh})\end{array}$ \\
\hline SmartLab & 38.12 & $\begin{array}{c}\text { Twitter } \\
\text { @SmartLaCoffee }\end{array}$ & 49.19 & $\begin{array}{c}\text { Forecasting }+ \\
\text { (ambient light arch \& } \\
\text { UV hidden message) }\end{array}$ & 34.82 & $\begin{array}{c}\text { Forecasting } \\
\text { (ambient light arch \& } \\
\text { UV hidden message) }\end{array}$ & 35.52 \\
\hline S3Lab & 37.62 & $\begin{array}{c}\text { Twitter } \\
\text { @ProtoLabCoffee }\end{array}$ & 53.68 & WebSite - Historical & 78.61 & WebSite - Historical & 75.36 \\
\hline ProtoLab & 62.33 & $\begin{array}{c}\text { Twitter } \\
\text { @S3LabCoffee }\end{array}$ & 84.65 & $\begin{array}{l}\text { Physical bar-chart } \\
\text { comparative }\end{array}$ & 115.85 & $\begin{array}{l}\text { Physical bar-chart } \\
\text { comparative }\end{array}$ & 39.59 \\
\hline
\end{tabular}

Table 3 The fluctuation of coffees' energy cost for each laboratory during four weeks. The table presents the comparative, energy-wise, performance of each laboratory and the eco-feedback each was subjected to.
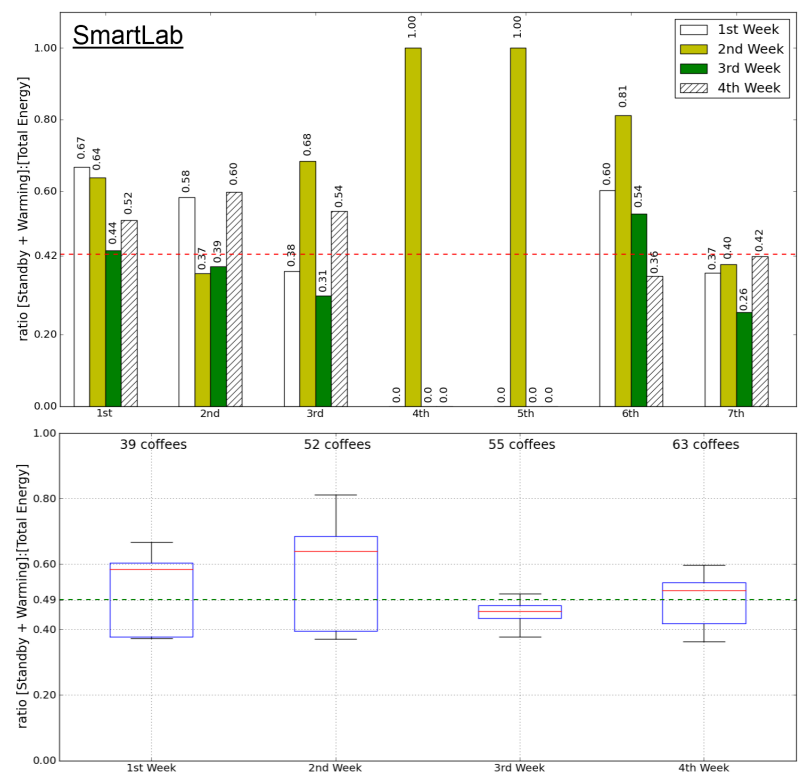

Fig. 5 a) The ratio of non-effective energy to the total along the days of the week ( $1^{\text {st }}$ plot-bar corresponds to Wednesday); b) A side-by-side box-plot to compare the distribution of the ratio of non-effective energy for each of the 4 weeks.

ratory, each of them with different consumption, makes the difference. The eco-aware coffee-maker performed real-time eco-feedback and decision-making assistance. Therefore we assume that the collaboration between people and the coffee-maker has boosted a reduction of energy waste and a more uniform device usage: 1) an increased motivation, i.e. reducing absent mindedness while maintaining comfort; 2) the aid offered by the coffee machine to enhance an appropriate device usage.

\subsection{Qualitative Results from Participants}

The summary from the post-trial questionnaire reveals that $65 \%$ of participants declared to have followed the interactions from the coffee-makers, while $76 \%$ asserted that they had reflected with work-colleagues about the energy updates. 4 over 6 of S3Lab's members, which was the laboratory with the worst response to the in- tervention, confessed to not have paid much attention to the experiment. Without showing any overview of the energy data to the participants, $50 \%$ opined that during the experiment the energy consumed was in general reduced, while $22 \%$ felt opposite. Interestingly, $73 \%$ of subjects attributed energy reductions to the coffeemaker and $82 \%$ of them said that the information reported increased their motivation.

The most promising finding was that in only two weeks receiving eco-feedback, $56 \%$ of the participants stated to have changed their way of using the coffeemaker. More important for us was to find that within such percentage, almost $80 \%$ belonged to the SmartLab, i.e. the laboratory where the eco-aware appliance was placed.

During the interview user C pointed out: "Now I wait until the message appears after preparing a coffee"; User D exhibits his awareness when stated: "I quickly switch it off [the coffee-maker] to not to see the amount of wasted energy increased".

Surprisingly, one of the commentaries received could glimpse a hint of behaviour change, even if it is known that such a short intervention of one month should be not enough to prompt it as discussed by Froehlich et al. (2010). This comment was extracted from the interview with user E:

"If I look around and I see somebody that is about to prepare a coffee after me, I leave the device on."

\subsubsection{Was the Eco-aware Coffee-maker Considered as a Teammate?}

Regarding the Likert questionnaire in the second part of the post-trial interview, we devoted 5 out of 18 items to get insights about the team relationship between the eco-aware coffee-maker and the people that interacted with it. The goal was to assess whether the end-users exhibit greater encouragement to save energy if the eco-aware everyday coffee-maker pursues the same goal and actively collaborates to attain it. A similar study 
was conducted by Nass et al. (1996). With an alpha value of $0.5 * 10^{-3}$ we got a statistical difference between the answers of the group that interacted with the ecoaware coffee-maker (SmartLab) regarding the other two groups (ProtoLab and S3Lab) $\chi^{2}(\mathrm{df}=2 ; \mathrm{N}=20)=17.5201$, $p=0.1569 * 10^{-3}$. The results are not determinant because of the length of the sample, but they shed light about the different perception that have the people who interacted with the eco-aware appliance in comparison with those who only received eco-feedback.

All the subject of the SmartLab declared to feel the coffee-maker as vital unit to save energy. When asked about the role of the coffee-maker in the group, $65 \%$ of the respondents attributed a role of authority and accordingly they heed their advices to save energy.

Interdependence cues, i.e. whether the participants exhibited any affiliation degree with the appliance to save energy, were also evaluated among the SmartLab workers. In this construct we got an agreement of the $76,47 \%$ in the 3 items out 18 that were evaluated. That means that the subjects under interdependence conditions were found to be more cooperative and open to influence from the eco-aware coffee-machine, similarly as Nass et al. (1996) evidenced in their work.

\section{Discussion}

In the current state, the methodology presented to transform the electric coffee makers into energy efficient devices would be extrapolate to the group of appliances which are shared among several people and which present the same kind of operating mode (e.g kettles, beamers, printers or public touch-screens, to cite some). Thus, devices which feature standby and ON-OFF modes with a high energy demanding boot-start. However, we recognise that in order to translate the devised machine learning algorithm to other appliances, such as those previously commented, different variables that were not applied in the current approach should be taken into account. Following, two examples where other variables are pivotal to attain the best of energy efficiency are illustrated: beamer projectors are not as frequently used as coffee makers in workplaces but they are in academic conferences. Therefore, the context is relevant. The same applies to the frequency of use of some devices when different weather conditions occurs. Other interesting example are printers. These devices are not always needed urgently as the coffee machines are, i.e. we usually can delay our documents to be printed, but is hard to delay a cup of coffee in a peak of work. Therefore, immediacy and comfort should be incorporated in the theoretical algorithms. This latter example is very relevant to the next steps of research with the coffee makers. We have realised that in many cases, the best strategy to reduce energy consumption it is not to motivate people to leave the device on or off, but ideally would be trying to change their habits to group the coffee intakes in the same time-slots or even better, to prepare them at the same time. Such strategy present some matching with the 'sharing economy' concept. That is, doing the things together help to save money and in this case, also to reduce $\mathrm{CO}_{2}$ emissions. In this supposed scenario where people prepare the coffee together, they would be also motivated to share the microwave to heat the milk, which in turns this action will save more energy. Although some academics could estimate that it is simplistic to just focus the efficiency approach to one appliance, we argue that whatever minimal energy reduction achieved with these devices will have a significant impact in terms of energy. The stock of electric coffee machines in EU in 2009 was estimated to be 100 million units (whereas a estimation of coffee machines in work-offices attained 30 million), consuming 17000 million of kWh per year (Bush et al. (2009)). Moreover, this tendency is prone to continue increasing and we have witnessed during our research that there is not a huge difference in terms of energy efficiency between old-fashioned models and new ones.

\section{Conclusions}

The goal of the presented work was to test whether is feasible to save energy in shared places through persuasive eco-aware everyday things powered by soft computing techniques managed in a Cloud service. We placed three capsule coffee machines in three different workplace locations during one month. The coffee machines were augmented to provide different means of eco-feedback to their users (from simple dashboard-based feedback to smart eco-aware-like feedback).

The field-study has yielded promising results for further research. The eco-aware appliance contributed to make workers become more energy consumption aware than they were at the beginning of the experiment. The group that jointly collaborated with the eco-aware coffee-maker was found to be the most efficient in energy terms. During the time the three different ecofeedback were provided, the other two groups wasted more than $200 \%$ of energy than the consumed by the eco-aware coffee-maker group. Likewise, this latter group attributed interdependence cues to the device and showed to be more open to influence from the advices of such eco-aware thing.

The study has presented a set of empirical evidences about how people and eco-aware things can perform joint actions to reduce energy consumption in common 
spaces as green-teammates. The findings have shed light on the following points: 1) web-based techniques, like dashboards, make difficult for users to correlate their actions with the energy associated to them. 2) simple informative eco-feedback is not always enough to know in real-time whether the action that the people in common spaces are performing is "environmentally adequate" or not; and 3) the combination of soft computing techniques and persuasive eco-feedback is an effective approach that worth continuing in order to explore how to reduce energy wastage at shared common spaces.

We are currently working in a longitudinal experiment with a wider population and with different background than the population used in the presented work (i.e., $N=20$ ). The goal will be to assess the statistical validity of our hypotheses and to strengthen the external validity of our results. We plan also to improve the forecasting operations. Although ARIMA has performed as the most accurate method with respect to three ANN configurations (see APENDIX A), we reckon that artificial neural networks can eventually yield better forecasting. Further, ANNs may exhibit lower power consumption during the forecasting operation, i.e. once trained, ANNs are more lightweight than ARIMA to be embedded in a micro-electronic platform, e.g. Arduino. The idea is to reduce the number of interactions between the eco-adaptor and the Cloud-service to keep as simple and efficient their operation as possible. Finally, we are willing to demonstrate that the design of the eco-adaptor is Carbon neutral, i.e. the $\mathrm{CO}_{2}$ footprint introduced by the electronic platform has always to be offset by the energy savings one obtains from using it. Moreover, further work should also attempt to prove that behaviour change is achieved. This is, users in different shared environments and without cues to behave responsibly still behave better, since they have been eco-educated by the eco-aware appliances.

Acknowledgements The authors are very grateful to the University of Deusto for the financial support to their PhD. studies and also to the project Future Internet II (IE11-316) supported by the Basque Government.

\section{APENDIX A}

ANNs as a soft computing technique are widely used as forecasting models in many areas. ANNs are datadriven, self-adaptive methods with few prior assumptions. They are also good predictors with the ability to make generalised observations from the results learnt from original data, thereby permitting correct inference of the latent part of the population. The wide use of
ANNs is due to their very efficient performance in solving nonlinear problems including those in real world. This is in contrast to ARIMA, which assume that the series are generated from linear processes and as a result might be inappropriate for most real-world problems that are nonlinear (Ariyo Adebiyi et al. (2014)). Although ANNs have provided competitive results when compared with ARIMA models (Khashei and Bijari (2010)), we have evidenced that in our specific case, the ARIMA-based model performs better when using the Mean Absolute Error (MAE), Root Mean Squared Error (RMSE), Mean Absolute Scaled Error (MASE) and Mean Absolute Percentage Error (MAPE) comparative metrics.

\section{ANNs in a Nutshell}

An ANN is an interconnected group of nodes able to approximate a functional relationship between input and output variables in a certain domain of interest. Using the analogy with human neural networks, the nodes, that compose the ANN, are said neurons and the directed edges which communicate them are called synapses. The neurons are organised in layers which are usually fully connected by synapses. Each of the synapses is attached with a weight indicating the effect of the corresponding neuron in the whole model. The data pass through the neural network as signals. They are first processed by the so-called integration function combining all incoming signals (usually a summation); and second, they are processed by the so-called activation function obtaining the output of the neuron. A neural network with zero hidden layers is a linear expansion. The general model of a neural network usually has three layers (one is the hidden layer) with a single output. It is represented by this equation:

$o(x)=f\left(w_{0}+\sum_{j=1}^{q} w_{j} \cdot f\left(w_{0 j}+\sum_{i=1}^{n} w_{i j} x_{i}\right)\right)$

where $w_{i j}(\mathrm{i}=1,2, . ., \mathrm{n} ; \mathrm{j}=1,2, . . \mathrm{q})$ and $w_{j}$ are the weights of synapses; $n$ is the number of input nodes and $q$ is the number hidden nodes; $f$ is the activation function. The definition of an ANN predictive model consists in determining the weights that provide the best fitting of the real data through usage of learning algorithms (Khashei and Bijari (2010)).

\section{ANN's Topology Selection}

This process encompasses the selection of the number of hidden layers, as well as the number of input, hidden and output neurons in each layer. As the model creation is driven by the data, we overview the shape of our time 
series. Our dataset which is openly available ${ }^{6}$ consisted of: 1) the training-set with the number of coffees prepared throughout each of the one-hour slots (starting at 7.00 a.m. and ending at 7.00 p.m) in 18 working days (weekends excluded). 2) The test-set which is the same type of data corresponding to the 5 consecutive working days; 3) 5 more working days of real data to test the models' performance.

Taking into account that one hidden layer is sufficient to model any piece-wise continuous function as stated by Hornik et al. (1989), we have chosen to use only one hidden layer in our model. Regarding the input layer, we have modelled the neural network with five input variables (i.e. five input neurons) that represent the number of coffees counted in the same hour slot along five consecutive days (from Monday to Friday). In the output layer we have decided to use only one neuron. It represents the number of predicted coffees that are prone to be prepared in the same hour-slot of those of the input nodes.

The idea of the proposed model is to feed it with a window of 5 working days of data (i.e. 12 vectors of 5 values each) to obtain the next-day forecast (i.e. a 12 values vector), slide the window forthward one day, including the previous predicted day, and perform again the prediction (See Figure 6). To clarify, if we feed the model with 5 values corresponding with the coffees prepared in one hour slot from Monday to Friday, we will expect to obtain the forecasted number of coffees from the next Monday at the corresponding slot. If we do the same form Tuesday of the previous week until Monday of the current week, we will expect to obtain Tuesday's forecasted number of coffees. If we slide again we will obtain the Wednesday forecast. So we repeat this process until the whole week is predicted.

\section{ANN's Training phase}

To select the most accurate model in order to compare it with ARIMA, we tested three different training configurations with respect to the number of hidden neurons: 5:2:1 (i.e. two hidden neurons); 5:5:1 (i.e. five hidden neurons); and 5:10:1 (i.e. ten hidden neurons). The training of the network is performed applying resilient backpropagation as a learning algorithm. The training-set is composed by the coffees counted in each hour slot during eighteen working days. Therefore, we train the ANN with 36 input vectors of five values each (15 days) and another 36 output vector of one value each (3 days). In this phase, the ANN calculates

\footnotetext{
6 https://dl.dropboxusercontent.com/u/3163534/dataset\%20softcomputing.zip
}

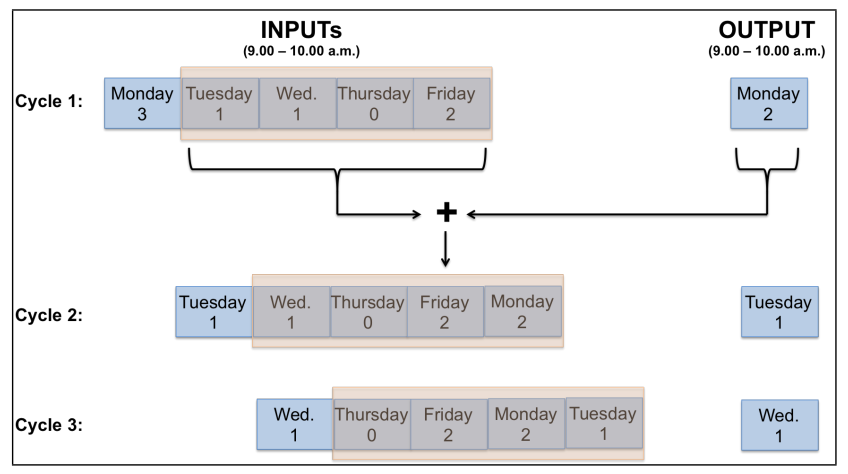

Fig. 6 The slidig window of 5 working days of data used to predict the next-day coffee intakes forecast. The process is repeated until the whole week is completed.

in an iterative manner the output for each given inputs, it measures the difference between the predicted and given output (i.e. the error) and it uses this error to modify the weights. All the three model configurations were respectively trained with 10 epochs by using the same training-set and learning algorithm.

\section{Testing Phase and Model Selection}

The last phase is the testing session. The test-set was composed by the coffees counted in one week. As the neural network model provides only one output, the execution of one test session is repeated by applying the sliding window approach of Figure 6.

To determine the best performing structure, we have calculated the different prediction errors for each of the models: RMSE, MAPE, MAE and MASE. They are shown in Table 2. For each neural network configuration these metrics were computed using the predicted values and the remaining five days of real data of our dataset.

In all evaluated indexes, ANN_2 (two hidden nodes) has smaller values than the other ANNs. However, assuming a Gaussian error distribution for our predictions, we have used the root-mean-squared-error (RMSE) to determine the best performing structure as was suggested by Chai and Draxler (2014). Table 2 shows that the RMSE value of testing error is 1.6278 for the configuration $5: 2: 1$, while it is 2.3614 and 2.0953 respectively for the neural networks' structures with five and ten hidden nodes. The smallest RMSE bares the best neural network configuration. Therefore, we can then conclude that for the time series data we had, the most accurate predictive model, when forecasting the weekly usage of a coffee machine, was a network with two nodes in the hidden layer. 


\begin{tabular}{lllll}
\hline & ARIMA & ANN_2 & ANN_5 & ANN_10 \\
\hline RMSE & 0.9708 & 1.6278 & 2.3614 & 2.0953 \\
MAPE & 0.8418 & 0.9413 & 1.0143 & 1.0447 \\
MAE & 1.0130 & 1.3142 & 1.8307 & 1.5076 \\
MASE & 0.3709 & 0.4812 & 0.6703 & 0.5520 \\
\hline
\end{tabular}

Table 4 Comparison of forecasting models. As it can be seen ARIMA outperforms the three configurations of ANN in all the error metrics.

\section{ARIMA outperforms ANN 5:2:1}

In a previous authors' publication we performed a forecasting model selection that better fitted with our specific time series data ${ }^{7}$.

The selected model was an $\operatorname{ARIMA}(3 ; 1 ; 1)(2 ; 0 ; 2)$ which is an ARIMA model with a seasonal part ( also called SARIMA). This model was chosen since it presented the smallest Akaike's Information Criterion (AIC) and because it did not present autocorrelations in the residuals. These two criterion demonstrated that the model provides an adequate fit to the data.

To compare the models' autocorrelated structure performance, we have confronted the ARIMA error measurements with regard to the three ANN configurations previously discussed. According to Table 2, ARIMA has the lowest percentage error in each of the metrics calculated. In view of the results, we consider that ARIMA is the a suitable forecasting technique for the coffee-maker appliance's usage.

\section{References}

A. Ariyo Adebiyi, A. Oluyinka Adewumi, C. Korede Ayo, Comparison of ARIMA and Artificial Neural Networks Models for Stock Price Prediction. Journal of Applied Mathematics (2014)

E. Arroyo, L. Bonanni, T. Selker, Waterbot: exploring feedback and persuasive techniques at the sink, in Proc. of CHI'05, 2005, pp. 631-639

G.E.P. Box, G. Jenkins, Time Series Analysis, Forecasting and Control, vol. Holden-Day, Incorporated 1990

L. Broms, C. Katzeff, et al., Coffee maker patterns and the design of energy feedback artefacts, in Proc. of DIS'10, vol. ACM, 2010, pp. 93-102

H. Brynjarsdóttir, M. Håkansson, E. Baumer, C. DiSalvo, P. Sengers, Pierce, James, Sustainably Unpersuaded: How Persuasion Narrows our Vision of Sustainability, in Proc. of CHI'12, vol. ACM, 2012, pp. 947-956

E. Bush, J. Nipkow, B. Josephy, S. Heutling, R. Griesshammer, Strategies to Enhance Energy Efficiency of Coffee Machines, in Proc. of EEDAL'09, 2009

T. Carbon, CarbonTrust: Employee Awareness and Office Energy Efficiency [Online] Accessed on 30/09/2014, 2013. http://tiny.cc/o5p5tw

7 A complete model description and the whole selection criteria was thoroughly described in López-de Armentia et al. (2014).
A.R. Carrico, M. Riemer, Motivating energy conservation in the workplace: An evaluation of the use of group-level feedback and peer education. Journal of Environmental Psychology 31(1), 1-13 (2011)

D. Casado-Mansilla, J. López-De-Armentia, P. Garaizar, D. López-De-Ipiña, To Switch the Coffee maker or Not: That is the Question to be Energy Efficient at Work, in Proc. of E.A. of CHI'14 (Publication pending) (ACM, ???, 2014)

T. Chai, R.R. Draxler, Root mean square error (rmse) or mean absolute error (mae)? arguments against avoiding rmse in the literature. Geoscientific Model Development $\mathbf{7}(3), 1247-1250$ (2014)

J. Chapman, Emotionally Durable Design: Objects, Experiences and Empathy, vol. Earthscan LLC 2005

M. Chetty, D. Tran, , R.E. Grinter, Getting to green: understanding resource consumption in the home, in Proc. of UbiComp'08, vol. ACM, 2008, pp. 242-251

E. Costanza, S.D. Ramchurn, N.R. Jennings, Understanding domestic energy consumption through interactive visualisation, in Proc. of UbiComp'12, vol. ACM, 2012, pp. 216-225

T. Crosbie, M. Houghton, Sustainability in the Workplace, Technical report, Sustainability at Work, 2012

Daamen, Dancker D. L. , Staats, Henk, Wilke, Henk A. M., Engelen, Mirjam, Improving environmental behavior in companies:the effectiveness of tailored versus nontailored interventions. Journal of Environment and Behavior 33(2), 229-248 (2001)

S. Darby, The effectiveness of feedback on energy consumption: a review for DEFRA of the literature on metering, billing and direct displays., Technical report, Univ. of Oxford, 2006

J.M. Darley, B. Latane, Bystander intervention in emergencies: diffusion of responsibility. Journal of personality and social psychology 8, 277-281 (1968)

C. DiSalvo, P. Sengers, H. Brynjarsdttir, Mapping the Landscape of Sustainable HCI, in Proc. of (CHI'10), vol. ACM, 2010, pp. 1975-1984

Energy Efficiency and Renewable Energy, Energy efficiency trends in residential and commercial buildings, Technical report, U.S. DoE), 2008

EU-Comission, Directive 2008/98/EC of the European Parliament and of the Council of 19 November 2008 on waste and repealing certain Directives, Technical report, European Comission, 2008. http://bit.ly/RdWxOj

D. Farber, CNET news: Twitter hits 400 million tweets per day, mostly mobile [Online] Accessed on 30/09/2014, 2012. http://cnet.co/KHlg8q

C. Fischer, Feedback on household electricity consumption: a tool for saving energy? Journal of Energy efficiency 1(1), 79-104 (2008)

G. Fitzpatrick, G. Smith, Technology-enabled feedback on domestic energy consumption. IEEE Pervasive Computing, 37-44 (2009)

B.J. Fogg, Persuasive Technology: Using Computers to Change What We Think and Do, vol. 18 2003, p. 283

B.J. Fogg, A Behavior model for Persuasive Design, in Proc. of PERSUASIVE'09, vol. Springer-Verlag, 2009, pp. 401407

D. Foster, S. Lawson, J. Wardman, M. Blythe, , C. Linehan, "Watts in it for me?": design implications for implementing effective energy interventions in organisations, in Proc. of CHI'12, vol. ACM, 2012, pp. 2357-2366. ISBN 978-1-4503-1015-4

D. Foster, S. Lawson, M. Blythe, P. Cairns, Wattsup?: Motivating Reductions in Domestic Energy Consumption Us- 
ing Social Networks, in Proc. NordiCHI'10, vol. ACM, 2010, pp. 178-187

J. Froehlich, L. Findlater, J. Landay, The Design of Ecofeedback Technology, in Proc. of CHI'10, vol. ACM, 2010, pp. 1999-2008

A. Gustafsson, M. Gyllenswrd, The power-aware cord: energy awareness through ambient information display, in $E A$ of CHI'05, vol. ACM, 2005, pp. 1423-1426

M.S. Horn, P. Davis, A.K. Hubbard, D. Keifert, Z.A. Leong, I.C. Olson, Learning sustainability: families, learning, and next-generation eco-feedback technology, in Proc. of IDC'11. IDC '11, vol. ACM, 2011, pp. 161-164

K. Hornik, M. Stinchcombe, H. White, Multilayer Feedforward Networks Are Universal Approximators. Journal of Neural Networks 2, 359-366 (1989)

M. Jahn, T. Schwartz, J. Simon, , M. Jentsch, EnergyPULSE: tracking sustainable behavior in office environments, in Proc. of e-Energy'11, vol. ACM, 2011, pp. 87-96

L. Jönsson, L. Broms, C. Katzeff, Watt-Lite: energy statistics made tangible, in Proc. of DIS'10, vol. ACM, 2010, pp. 240-243

M. Khashei, M. Bijari, An artificial neural network (p,d,q) model for timeseries forecasting. Expert Syst. Appl. 37, 479-489 (2010)

J. López-de-Armentia, D. Casado-Mansilla, D. López-deIpiña, Reducing energy waste through eco-aware everyday things. journal of Mobile Information Systems 10 (2014)

C. Nass, B.J. Fogg, , Y. Moon, Can computers be teammates? Int. J. Hum.-Comput. Stud. 45(6), 669-678 (1996)

J. Pierce, E. Paulos, Beyond Energy Monitors: Interaction, Energy, and Emerging Energy Systems, in Proc. of CHI'12, vol. ACM, 2012, pp. 665-674

R, R-Core Team, R: A Language and Environment for Statistical Computing. R Foundation for Statistical Computing, Vienna, Austria, 2013. ISBN 3-900051-07-0. http://www.R-project.org/

A. Schmidt, H.-W. Gellersen, M. Beigl, Matching information and ambient media, in Cooperative Buildings. Integrating Information, Organizations, and Architecture, vol. Springer vol. Springer 1999, pp. 140-149

T. Schwartz, M. Betz, L. Ramirez, G. Stevens, Sustainable Energy Practices at Work: Understanding the role of Workers in Energy Conservation, in Proc. of NordiCHI'10, vol. ACM, 2010, pp. 452-462

F.W. Siero, A.B. Bakker, G.B. Dekker, , M.T.C. Van-denBurg, Changing organizational energy consumption behaviour through comparative feedback. Journal of Environmental Psychology 16(3), 235-246 (1996)

M. Starik, A.A. Marcus, Introduction to the special research forum on the management of organizations in the natural environment. Journal of Academy of Management 43(4) (2000)

Thieme, A., Comber, R., Miebach, J. , Weeden, J. , Kraemer, N. , Lawson, S., Olivier, P. , We've bin Watching you": Designing for Reflection and Social Persuasion to Promote Sustainable Lifestyles, in Proc. of CHI'12, vol. ACM, 2012, pp. 2337-2346

D. Ventura, D. Casado-Mansilla, J. López-de-Armentia, P. Garaizar, V. Catania, D. López-de-Ipiña, ARIIMA: A Real IoT Implementation of a Machine-learning Architecture for reducing energy consumption, in Proc. of UCAmI'14, vol. LNCS, 2014

R.A. Winett, M.S. Neale, H.C. Grier, Effects of selfmonitoring and Feedback on Residential Electricity Consumption. Journal of Applied Behavior Analysis 12(2),
173-184 (1979)

R. Yang, M.W. Newman, Learning from a Learning Thermostat: Lessons for Intelligent Systems for the Home, in Proc. of UbiComp'13, vol. ACM, 2013, pp. 93-102

R. Yun, P. Scupelli, A. Aziz, V. Loftness, Sustainability in the workplace: nine intervention techniques for behavior change, in Proc. of PERSUASIVE'13, vol. SpringerVerlag vol. Springer-Verlag 2013a, pp. 253-265

R. Yun, B. Lasternas, A. Aziz, V. Loftness, P. Scupelli, A. Rowe, R. Kothari, F. Marion, J. Zhao, Toward the design of a dashboard to promote environmentally sustainable behavior among office workers, in Proc. of PERSUASIVE'13, vol. Springer-Verlag, 2013b, pp. 246-252 